\title{
Video Article \\ Chemical Synthesis of Porous Barium Titanate Thin Film and Thermal Stabilization of Ferroelectric Phase by Porosity-Induced Strain
}

\author{
Norihiro Suzuki ${ }^{1,2}$, Minoru Osada ${ }^{3}$, Motasim Billah ${ }^{3,4}$, Yoshio Bando ${ }^{3,4}$, Yusuke Yamauchi ${ }^{5,6}$, Shahriar A. Hossain ${ }^{3,4}$ \\ ${ }^{1}$ Research Institute for Science and Technology (RIST), Tokyo University of Science (TUS) \\ ${ }^{2}$ International Center for Young Scientists (ICYS), National Institute for Materials Science (NIMS) \\ ${ }^{3}$ International Center for Materials Nanoarchitectonics (MANA), National Institute for Materials Science (NIMS) \\ ${ }^{4}$ Australian Institute for Innovative Materials (AIIM), University of Wollongong \\ ${ }^{5}$ School of Chemical Engineering, Australian Institute for Bioengineering and Nanotechnology (AIBN), University of Queensland \\ ${ }^{6}$ Department of Plant \& Environmental New Resources, Kyung Hee University
}

Correspondence to: Norihiro Suzuki at suzuki.norihiro@rs.tus.ac.jp

URL: https://www.jove.com/video/57441

DOI: doi:10.3791/57441

Keywords: Retraction, Issue 133, Barium Titanate, Surfactant-Assisted Sol-Gel Method, Porous Thin Film, Ferroelectric Phase, Thermal Stability, Anisotorpic Strain

Date Published: 3/27/2018

Citation: Suzuki, N., Osada, M., Billah, M., Bando, Y., Yamauchi, Y., Hossain, S.A. Chemical Synthesis of Porous Barium Titanate Thin Film and Thermal Stabilization of Ferroelectric Phase by Porosity-Induced Strain. J. Vis. Exp. (133), e57441, doi:10.3791/57441 (2018).

\section{Abstract}

Barium titanate $\left(\mathrm{BaTiO}_{3}\right.$, hereafter BT) is an established ferroelectric material first discovered in the 1940s and still widely used because of its well-balanced ferroelectricity, piezoelectricity, and dielectric constant. In addition, BT does not contain any toxic elements. Therefore, it is considered to be an eco-friendly material, which has attracted considerable interest as a replacement for lead zirconate titanate (PZT). However, bulk BT loses its ferroelectricity at approximately $130{ }^{\circ} \mathrm{C}$, thus, it cannot be used at high temperatures. Because of the growing demand for hightemperature ferroelectric materials, it is important to enhance the thermal stability of ferroelectricity in BT. In previous studies, strain originating from the lattice mismatch at hetero-interfaces has been used. However, the sample preparation in this approach requires complicated and expensive physical processes, which are undesirable for practical applications.

In this study, we propose a chemical synthesis of a porous material as an alternative means of introducing strain. We synthesized a porous BT thin film using a surfactant-assisted sol-gel method, in which self-assembled amphipathic surfactant micelles were used as an organic template. Through a series of studies, we clarified that the introduction of pores had a similar effect on distorting the BT crystal lattice, to that of a heterointerface, leading to the enhancement and stabilization of ferroelectricity. Owing to its simplicity and cost effectiveness, this fabrication process has considerable advantages over conventional methods.

\section{Video Link}

The video component of this article can be found at https://www.jove.com/video/57441/

\section{Introduction}

Barium titanate $\left(\mathrm{BaTiO}_{3}\right.$, hereafter $\left.\mathrm{BT}\right)$ is a typical perovskite-type ferroelectric material. Although its ferroelectric properties were discovered in the 1940s, it is still widely used today because of its well-balanced ferroelectric and piezoelectric responses and favorable dielectric constant. Furthermore, because BT is a lead-free, eco-friendly material, it has attracted great interest as a replacement for lead zirconate titanate (PZT). At room temperature, the crystal phase of BT is tetragonal, where the ratio of $c$ and a lattice parameters $(c / a)$ is not equal to 1 . In the tetragonal phase, the BT lattice is slightly elongated toward the $c$-axis and cations $\left(\mathrm{Ba}^{2+}, \mathrm{Ti}^{4+}\right)$ and anions $\left(\mathrm{O}^{2-}\right)$ are displaced in opposite directions. This displacement results in the spontaneous polarization of BT. When the temperature increases to the Curie temperature $\left(T_{\mathrm{c}}\right)$, a phase transition to the cubic phase occurs. In the cubic phase of $B T$, which has $c / a=1$, the lattice distortion is relaxed, and its ferroelectricity is lost owing to the electrical neutrality originating from the inversion symmetry of the lattice. Recently, the use of high temperature ferroelectric materials has expanded. However, the $T_{\mathrm{c}}$ of $\mathrm{BT}$ is relatively low $\left(\sim 130^{\circ} \mathrm{C}\right)$ and bulk BT does not meet these demands.

To increase the $T_{\mathrm{c}}$ of $\mathrm{BT}$, the ferroelectric (tetragonal) phase has been stabilized by the application of strain at the hetero-interface. For example, Choi et al. enhanced the ferroelectricity of BT films epitaxially grown on $\mathrm{GdScO}_{3}(110)$ and $\mathrm{DyScO}_{3}(110)$ substrates through the use of biaxial compressive strain caused by lattice mismatch ${ }^{1}$. However, the increase of $T_{\mathrm{c}}$ is limited to very thin films (tens of nanometers thick) ${ }^{2,3}$, which is impractical for device applications.

To increase the BT film thickness while preventing strain relaxation, superlattice (periodic structure of very thin layers) and three-dimensional (3D) hetero-nanostructures have been developed. Harrington et al. synthesized a vertical mesostructure of $\mathrm{BT}_{\text {and }} \mathrm{Sm}_{2} \mathrm{O}_{3}$ and obtained a micrometer-scale-ordered thick film without strain relaxation. In this sample, spontaneous polarization is oriented perpendicular to the substrate owing to uniaxial expansion of the BT unit cell; thus, a large remnant polarization was maintained at high temperature (i.e., the $T_{\mathrm{c}}$ was greater 
than $\left.800^{\circ} \mathrm{C}\right)^{4}$. The obtained properties were satisfactory; however, a complicated and expensive physical process (pulsed-laser deposition) was required for the fabrication, which is a drawback for practical applications.

As an alternative facile and inexpensive fabrication process, we have proposed the chemical synthesis of 3D nanocomposites by the introduction of a precursor solution of BT into pores of a porous strontium titanate $\left(\mathrm{SrTiO}_{3}\right.$, hereafter ST) thin film ${ }^{5}$. In the study, the porous ST thin film was synthesized by a surfactant-assisted sol-gel method, in which the self-assembly of amphipathic surfactant micelles was used as an organic template $^{6,7}$. The method is illustrated schematically in Figure 1. Because the obtained ST thin film has a complex 3D porous structure with a large surface area, strain at the BT/ST hetero-interface is introduced into the nanocomposite, leading to the stabilization of the ferroelectric phase of BT (the $T_{\mathrm{c}}$ of ST/BT nanocomposite reached $230^{\circ} \mathrm{C}$ ).

We hypothesized that porosity could directly introduce strain in BT and enhance the thermal stability of ferroelectric properties. In this study, we used a surfactant-assisted sol-gel method to fabricate porous BT and scrutinize the pore-induced strain. In addition, we compared the thermal stability between porous BT and nonporous bulk BT. We found that the introduced pores induced an anisotropic strain, which elongated the BT crystal lattice. This effect might be favorable for stabilizing the ferroelectric phase. Because the synthesis process used here is very simple, it has advantages over conventional physical processes for 3D hetero-nanostructures.

\section{Protocol}

\section{Preparation of Precursor Solution}

1. Dissolve $50 \mathrm{mg}$ of diblock copolymer PS(18000)-b-PEO(7500) in $1.5 \mathrm{~mL}$ of tetrahydrofuran at $40{ }^{\circ} \mathrm{C}$. Cool the polymer solution to room temperature (RT).

2. Dissolve $127.7 \mathrm{mg}$ of barium acetate in $830 \mu \mathrm{L}$ of acetic acid by stirring at $40{ }^{\circ} \mathrm{C}$ for $5 \mathrm{~min}$. Cool the barium acetate solution to RT. Add 170 $\mathrm{mg}$ of titanium butoxide to the barium acetate solution and stir the reaction mixture for $1 \mathrm{~min}$.

3. Add the polymer solution to the barium acetate solution dropwise.

\section{Synthesis of Mesoporous Barium Titanate Thin Film}

1. Set the $\mathrm{Si} / \mathrm{SiO}_{\times} / \mathrm{Ti} / \mathrm{Pt}$ substrate $(2 \mathrm{~cm} \times 2 \mathrm{~cm})$ on the stage of a spin-coater and drop the prepared precursor solution to entirely cover the substrate.

NOTE: The film thickness of $\mathrm{SiO}_{x}, \mathrm{Ti}$, and Pt layers were approximately $1.6,40$, and $150 \mathrm{~nm}$, respectively.

2. Spin the $\mathrm{Si} / \mathrm{SiO}_{\mathrm{x}} / \mathrm{Ti} / \mathrm{Pt}$ substrate at $500 \mathrm{rpm}$ for $5 \mathrm{~s}$ (1st step), and then $3,000 \mathrm{rpm}$ for $30 \mathrm{~s}$ (2nd step), successively.

3. Place the as-prepared film on a hot plate and heat it to $120^{\circ} \mathrm{C}$ for $5 \mathrm{~min}$ for aging, then allow it to cool to room temperature (RT) naturally.

4. Place the annealed film in a muffle furnace and calcine in air at $800^{\circ} \mathrm{C}$ for $10 \mathrm{~min}$ with a ramp rate of $1{ }^{\circ} \mathrm{C} / \mathrm{min}$ (for both heating and cooling).

\section{Characterization}

1. Morphological and crystallographic characterization

1. Scanning electron microscopy (SEM) measurement

1. Put the sample on the sample stage and cover the corners with carbon tapes to fix the sample. Adjust the height of the sample holder.

2. Set the sample holder in a loading rod and insert it to the SEM. Position the sample holder in the home position at a working distance of $8 \mathrm{~mm}$.

3. Set accelerating voltage and emission current to $5 \mathrm{KV}$ and $10 \mathrm{~mA}$ and generate an electron beam. Display the entire image of the sample at low magnification. Move the stage to show a region of interest (ROI) and focus on the image.

4. Increase the magnification to 50,000X. Focus on the pores and observe the porous morphology. When a suitable image is observed, save a picture.

2. Transmission electron microscopy (TEM) measurement

1. Prepare a cross-sectional specimen prior to the TEM measurement as described in step 3.3.1.

2. Set the prepared specimen in the sample holder. Set accelerating voltage to $300 \mathrm{KV}$ and generate an electron beam. Insert the holder to the TEM.

3. Display the entire image of the sample at low magnification. Move the stage to show ROI and focus on the image.

4. Increase the magnification to $250,000 X$. Focus on the sample and observe the porous morphology of the synthesized thin film ( $200 \mathrm{~nm}$ in thickness). When a suitable image is observed, save a picture.

3. Wide-angle X-ray diffraction (XRD) measurement

1. Set up the X-ray diffractometer equipped with a Cu Ka source.

2. Put the sample on the center of the sample stage.

3. Adjust the Z-axis (i.e., height position) of the stage in which the sample blocks the half of incident X-ray. Then, adjust the $\omega$-axis (i.e., grazing angle) to make the sample surface parallel to the X-ray.

4. Repeat step 3.1.3.3 until the sample stage position becomes suitable (i.e., the sample surface is located at the center of X-ray beam and parallel to the $\mathrm{X}$-ray).

5. Fix $\omega$ to a small angle $\left(\right.$ e.g., $0.5^{\circ}$ ), and then scan $2 \theta$ (i.e., detector angle) from $20^{\circ}$ to $70^{\circ}$ with a rate of $1^{\circ} / \mathrm{min}$. Save the data after the measurement. 
NOTE: To suppress the background signal from the substrate, incoming X-rays were irradiated at a very small grazing angle to the synthesized thin film's surface.

\section{Test for thermal stability of ferroelectric phase}

1. Turn on the Raman microscope and the operating computer, and then open the operating software LabSpec. Click Auto Calibration to calibrate the machine.

2. Set a glass slide on a heating stage, and then put the synthesized film on the glass. Close the cover of the stage.

3. Heat the sample with a heating stage using a ramp ratio of $15^{\circ} \mathrm{C} / \mathrm{min}$. When the temperature reaches the target temperature, set the heating stage to keep this temperature and wait a few minutes.

NOTE: In this study, we selected RT and $50,75,100,110,120,125,130,135,140$, and $150{ }^{\circ} \mathrm{C}$ for the bulk BT, which has a Curie temperature of approximately $130^{\circ} \mathrm{C}$. We selected temperatures of RT and $75,125,175,225,275,325,375,425,475$, and $525^{\circ} \mathrm{C}$ for the mesoporous BT thin film, for which the Curie temperature was evaluated to be approximately $470{ }^{\circ} \mathrm{C}$ from a previous study ${ }^{8}$.

4. After the temperature stabilizes, measure Raman spectra at various temperatures with a confocal Raman microscope using a 300$\mu \mathrm{m}$ confocal hole and a 532-nm laser (10 mW at sample) for excitation. Click the Video icon to display the observed image, and then focus on the image. Set acquisition time and accumulations to $100 \mathrm{~s}$ and 3, respectively, and then click Measure icon to start the measurement. Save the data after the measurement.

5. Continue the measurements until the spectrum changes (i.e., the peaks assignable to the ferroelectric phase disappears). NOTE: Measurements were conducted at three points and the obtained spectra were averaged.

\section{Visualization of the strain}

1. Prepare a cross-sectional specimen from the synthesized thin film by a micro sampling method using a focused ion beam for peripheral milling, bottom and micro-bridge cutting, and thinning of the specimen. The specimen's size should be approximately $20 \mu \mathrm{m}$ in length and $4 \mu \mathrm{m}$ in thickness.

2. Measure high-resolution transmission electron microscopy (HR-TEM) images $(2,000,000 \mathrm{X})$ at convex and concave surfaces induced by the porosity.

3. Select an area $(512 \times 512$ pixels) for fast Fourier transform (FFT), and calculate the FFT pattern. Estimate the lattice spacing from the FFT pattern, and divide it by the strain-free lattice spacing to calculate the "deformation ratio".

4. Shift the FFT analysis region by 32 pixels, and repeat step 3.3.3. Continue this process until the whole area of the HR-TEM image is covered (in this study, 5,664 × 5,664 pixel $(162 \times 162$ points)). NOTE: The strain-free lattice spacing was estimated from the least distorted FFT image.

5. Set the color of regions based on the calculated deformation ratio to visualize the strain. Make the histograms by counting the deformation ratio.

NOTE: Analysis of the deformation in HR-TEM images was conducted with the software CryStMapp.

6. Create a detailed histogram using the software Igor Pro

NOTE: Because the histogram of the distortion obtained in step 3.3.5 is rough, a detailed histogram using the software Igor Pro is created.

1. Load the numerical data of the deformation ratio by selecting Data | Load Waves | Load General Text and save it as a wave with proper name.

NOTE: Igor Pro defines the object including a numerical array as a wave.

2. Change the saved wave as a matrix $(162 \times 162)$ by selecting Data | Change Wave Scaling

3. Display a wave as 2D image by selecting Image | New | Image Prot.

4. Select Analysis | Packages | Image Processing to show Image menu.

5. Select Image | ROI to show ROI panel. Select Start ROI Draw to select ROI. Draw the region on top of an image, and then select Finish ROI.

6. Select Save ROI Copy to create the mask to select the area for analysis as ROI_M_Mask wave.

7. Input "ImageHistogram/R=M_ROIMASK/S waveneame" in the command line of the command window to make a histogram. Use the name set in step 3.3.6.1 in wavename.

8. Input "display W_ImageHist" in the command line of the command window to show a histogram. Modify the graph, if needed.

\section{Representative Results}

The morphology of the obtained mesoporous BT thin film was examined by electron microscopy. A top-view SEM image confirmed the porous features of the synthesized BT thin film (Figure 2a). The morphological features in the depth direction were investigated with a cross-sectional TEM image (Figure $\mathbf{2 b}$ ). Large crystallites with diameters of several tens of nanometers were vertically stacked, and the gaps between these crystallites were pores. The estimated thickness of the BT thin film was approximately $200 \mathrm{~nm}$.

The crystallinity of the BT framework was examined by wide-angle XRD measurements. Very weak peaks of $\mathrm{BaCO}_{3}$ and $\mathrm{TiO}_{2}$ were detectable and prominent peaks assignable to BT crystals were clearly observed (Figure 3a). However, it was difficult to distinguish between the (ferroelectric) tetragonal and (paraelectric) cubic phases. This is because the XRD patterns of both phases are quite similar. The main difference is that the peak at $2 \theta=45^{\circ}$ for the cubic phase is split for the tetragonal phase. In this study, the detection of such splitting was difficult because the polycrystalline nature of the film broadened the peak width. Thus, to clarify the crystal phase of the thin film, its Raman spectrum was measured (Figure 3b). The Raman spectrum of a bulk single BT crystal at room temperature showed peaks centered at $275,305,515$, and 720 $\mathrm{cm}^{-1}$, which were assigned to $A_{1}(\mathrm{TO}), B_{1}+E(\mathrm{TO}+\mathrm{LO}), E(\mathrm{TO})+A_{1}(\mathrm{TO})$, and $E(\mathrm{LO})+A_{1}(\mathrm{LO})$ modes of the tetragonal phase ${ }^{9}$. In the spectrum of the porous $\mathrm{BT}$ thin film, although splitting of the $A_{1}(\mathrm{TO})$ mode occurred, the main features of the spectrum were retained. Thus, the synthesized porous BT thin film was tetragonal. 
The spatial distribution of the strain in the BT framework of the thin film was examined by the fast Fourier transform mapping (FFTM) method ${ }^{10}$. This method analyzes and visualizes tiny distortions in FFT patterns of high-resolution (HR)-TEM images. Figure 4 depicts HR-TEM images of areas of the thin film with convex and concave surfaces and the corresponding FFTM images. The FFTM image of the [1-10] direction in a convex area revealed that the outermost convex surface was slightly expanded, an arrangement which should cause lattice relaxation and weakens ferroelectricity. Conversely, the areas just below the surface were compressed, and the compressed areas were observed entirely inside the framework. This result is consistent with previous reports that indicate the surface of BT nanoparticles feature a paraelectric cubic phase, while the inner core is a ferroelectric tetragonal phase ${ }^{11,12}$. Within the BT framework, some expanded areas were also found, mainly at kinks and/or grain boundaries (Figure 4c). For concave areas, although the deformation of the outermost surface was not clearly observed, likely because the surface was polygonal rather than curved, compression within the framework was detected (Figure 4d). Conversely, the FFTM images of the [11-1] direction in both the convex and concave areas were unclear (Figure 4e, f), suggesting that there was little deformation of the BT unit cell in this direction.

To examine the deformation of the BT lattice more quantitatively, the degree of deformation was summarized in histograms (Figure 5). From these histograms, we determined the "deformation ratio", which is defined as the ratio of the distance between adjacent lattice spacing in the target and reference areas, as a measure of deformation. In the [11-1] direction, the histograms were centered at a deformation ratio of 1.00 and were nearly symmetrical for both convex and concave areas. This result indicates that there was little strain in the [11-1] direction, consistent with the results of FFTM mentioned above. Conversely, the histograms for the [1-10] direction contained marked peaks at a deformation ratio of approximately 0.99 , showing the area where compressive strain increased in the BT thin film.

The thermal stability of the ferroelectric tetragonal phase was examined from the temperature dependence of its Raman spectrum (Figure 6). In a bulk BT single crystal, the sharp peaks at 305 and $720 \mathrm{~cm}^{-1}$ disappeared at $140{ }^{\circ} \mathrm{C}$, which is consistent with the $T_{c}$ of bulk BT $\left(\sim 130{ }^{\circ} \mathrm{C}\right)$. Conversely, the peak at $710 \mathrm{~cm}^{-1}$ from the tetragonal phase remained at much higher temperatures, detectable up to $375{ }^{\circ} \mathrm{C}$ for the synthesized porous thin film.

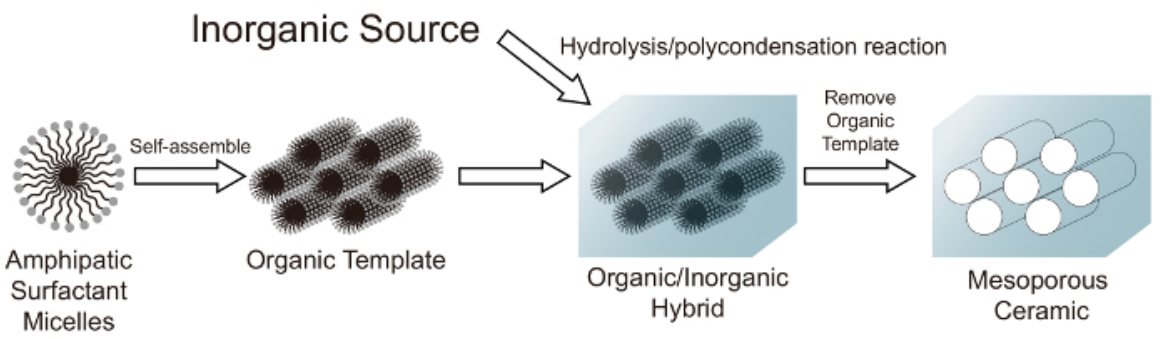

Figure 1: Schematic image of surfactant-assisted sol-gel method. Self-assembly of amphipathic surfactant micelles was used as a template. By combining the organic-template and inorganic sol, an organic/inorganic hybrid is created. Finally, calcination was conducted to create pores by removing the organic template and crystallizing the inorganic framework. Please click here to view a larger version of this figure.
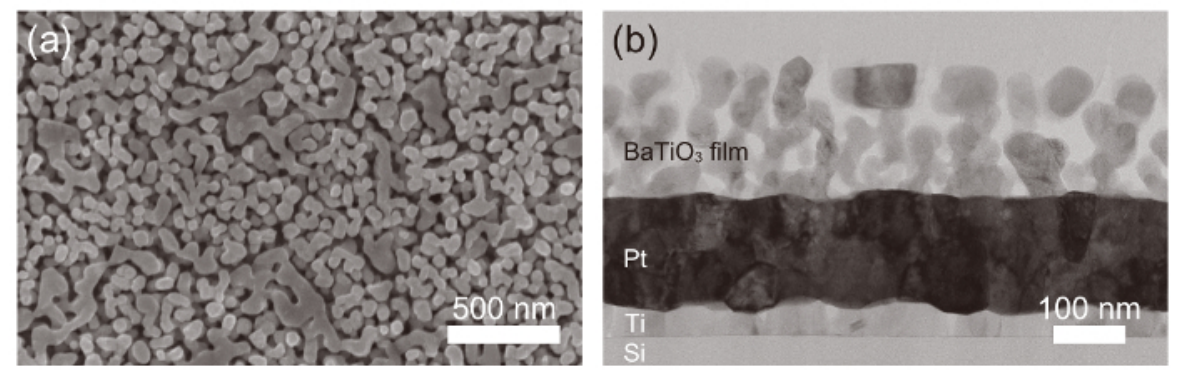

Figure 2: Electron microscope image of mesoporous BT thin film. (a) Top-view SEM and (b) cross-sectional TEM image. This figure has been modified from Suzuki, N. et al. ${ }^{13}$ in accordance with the Creative Commons Attribution (CC BY) license. Please click here to view a larger version of this figure. 

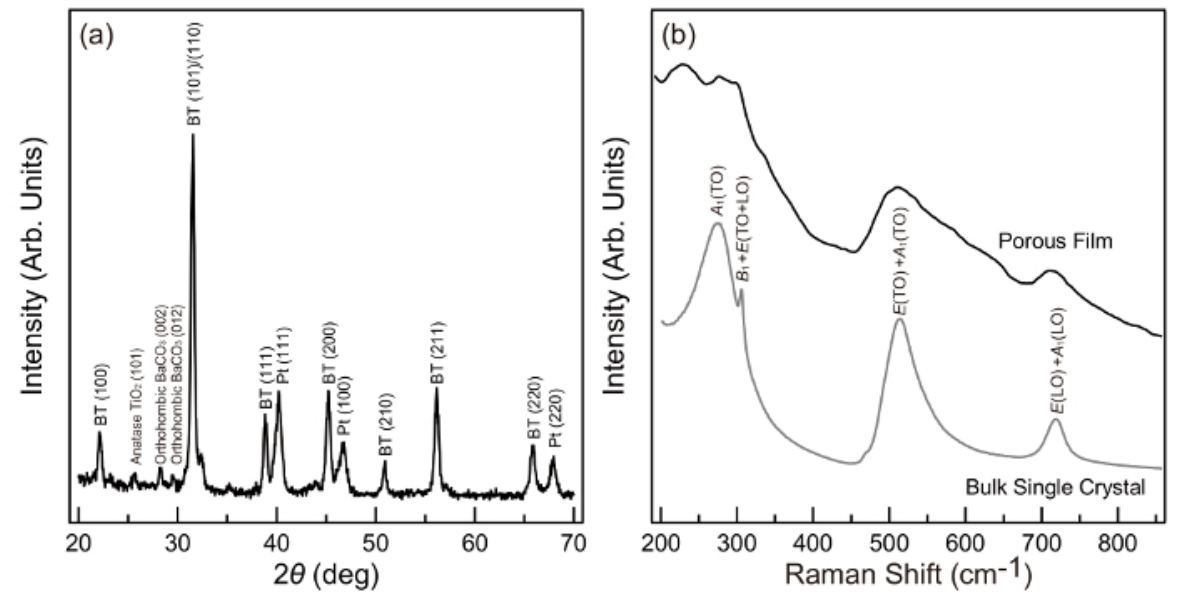

Figure 3: Spectroscopic data for the crystal phase of mesoporous BT thin film. (a) Wide-angle XRD pattern and (b) Raman spectrum of the synthesized porous BT thin film at room temperature. The spectrum of a bulk BT single crystal is also included for reference. This figure has been modified from Suzuki, N. et al. ${ }^{13}$ in accordance with the Creative Commons Attribution (CC BY) license. Please click here to view a larger version of this figure.
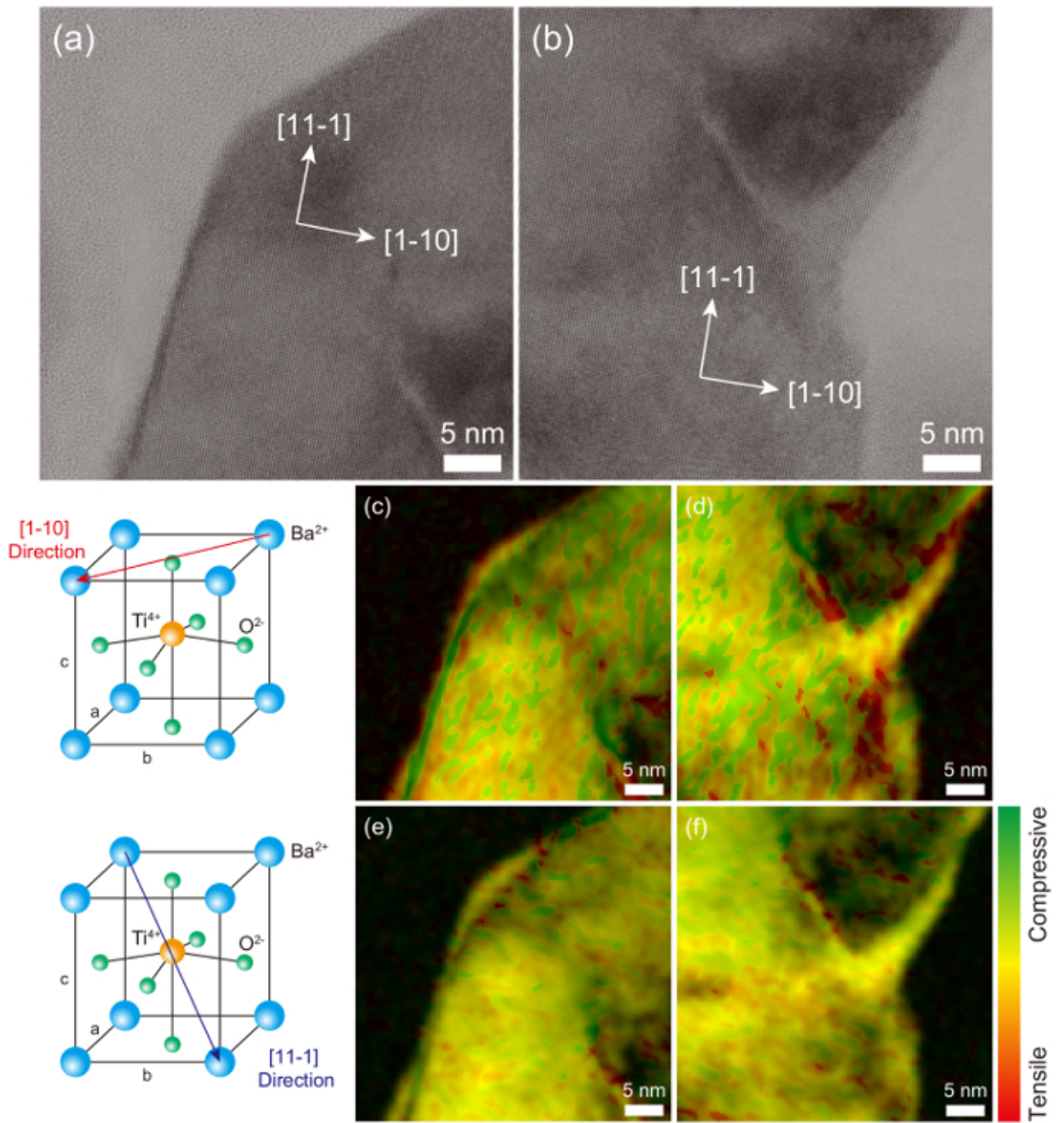

Figure 4: Visualization of porosity-induced strain. (a,b) High-resolution TEM images and (c-f) fast Fourier transform mapping (FFTM) images of $(\mathbf{a}, \mathbf{c}, \mathbf{e})$ convex and $(\mathbf{b}, \mathbf{d}, \mathbf{f})$ concave areas of the BT thin films. The orientations of the FFTM images are $(\mathbf{c}, \mathbf{d})[1-10]$ and $(\mathbf{e}, \mathbf{f})$ [11-1]. In the FFTM images, green and red areas represent regions where compressive and tensile strain are applied, respectively, while yellow indicates the reference area. The orientation used for the analysis is also included (left). This figure has been modified from Suzuki, N. et al. ${ }^{13}$ in accordance with the Creative Commons Attribution (CC BY) license. Please click here to view a larger version of this figure. 

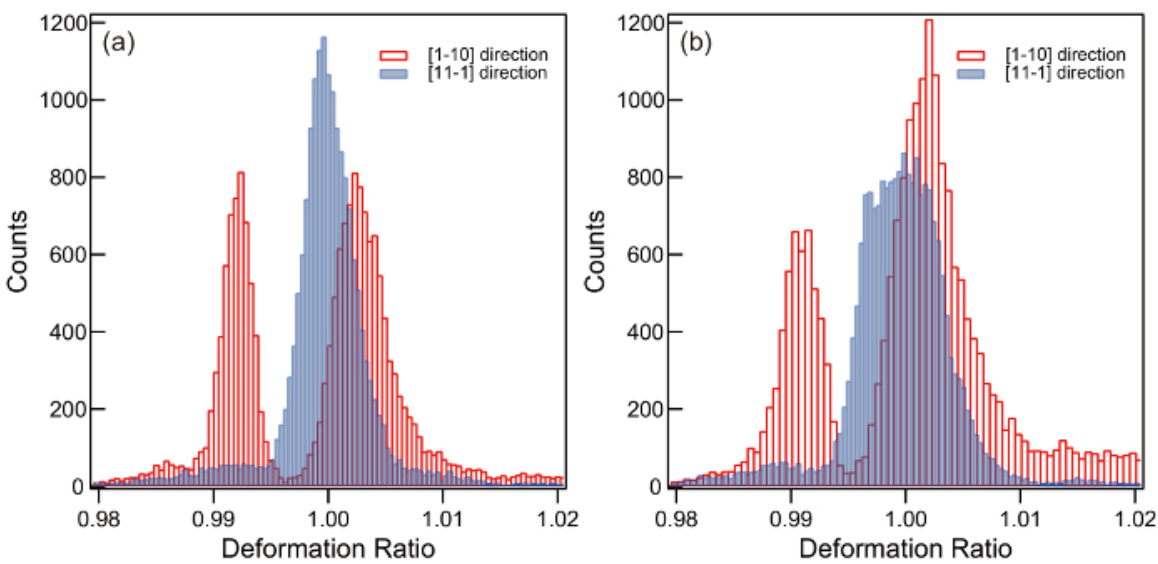

Figure 5: Analysis of porosity-induced strain. Histogram of distortion analyzed in (a) convex (Figure 4a, Figure 4c, and Figure 4e) and (b) concave (Figure $\mathbf{4 b}$, Figure $\mathbf{4 d}$, Figure $\mathbf{4 f}$ ) areas of the porous BT thin film. This figure has been modified from Suzuki, N. et al. ${ }^{13}$ in accordance with the Creative Commons Attribution (CC BY) license. Please click here to view a larger version of this figure.
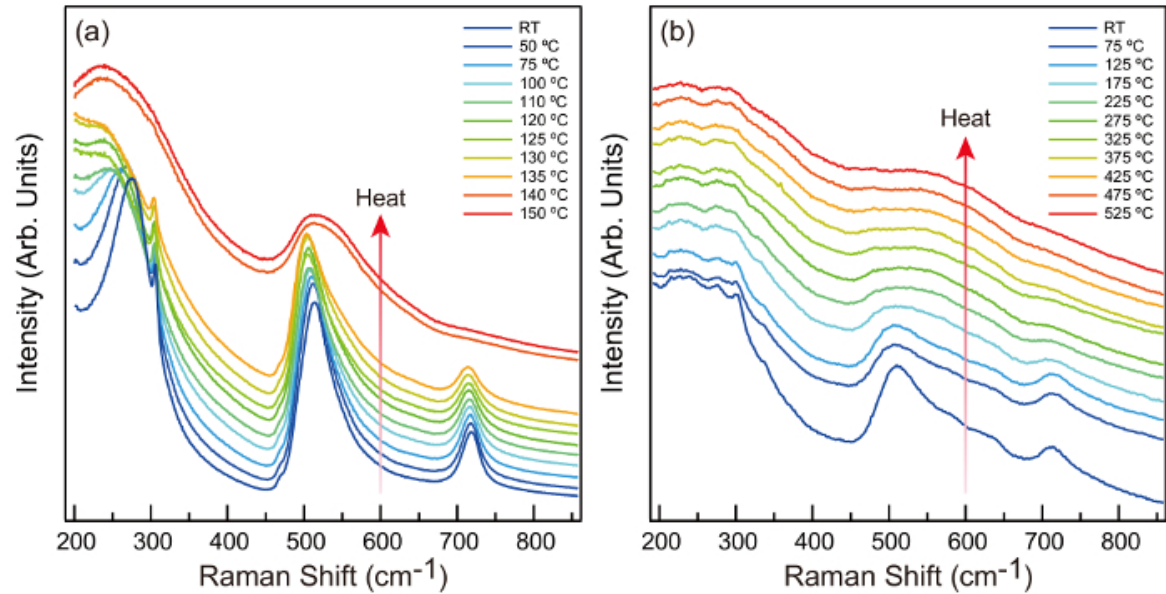

Figure 6: Thermal stability of ferroelectric phase. Temperature dependence of the Raman spectrum of (a) a bulk BT single crystal and (b) a synthesized porous BT thin film. This figure has been modified from Suzuki, N. et al. ${ }^{13}$ in accordance with the Creative Commons Attribution (CC BY) license. Please click here to view a larger version of this figure.

\section{Discussion}

The splitting of the $A_{1}(\mathrm{TO})$ mode in the Raman spectrum of a porous $\mathrm{BT}$ thin film (Figure $\mathbf{3 b}$ ) originates from compressive strain. This feature was clearly observed by the FFTM method (Figure 4) and its anisotropy in the [1-10] direction was determined from the histogram of distortion (Figure 5). Compressive strain along the [1-10] direction has a similar effect of inducing biaxial compressive strain in the (001) surface, which enhances the ferroelectricity in $\mathrm{BT}^{1}$. Porous driven anisotropic strain elongates the crystal lattice toward the $c$-axis, causing further dislocation of $\mathrm{Ti}^{4+}$ from the center of the lattice. This dislocation is expected to increase the electric dipole moment, which in turn enhances its ferro(piezo)electricity. Indeed, the piezoelectricity of a mesoporous BT film is superior to that of a non-porous film ${ }^{8}$.

The strain induced in the BT crystal lattice stabilizes the distorted tetragonal phase. Thus, the thermal stability of the lattice is expected to be enhanced. The Raman spectrum of the porous BT thin film showed the tetragonal phase origin peak (at $710 \mathrm{~cm}^{-1}$ ) remained visible until $375{ }^{\circ} \mathrm{C}$, although the peak gradually became weaker and broader (Figure $6 \mathbf{b}$ ). This trend was similar to that found in a previous study, in which the $T_{\mathrm{c}}$ was estimated to be $470{ }^{\circ} \mathrm{C}^{8}$. Thus, we confirmed the assumption that the pore-driven strain in the BT thin film effectively thermally stabilized the tetragonal phase.

Through this study, we clarified that pore-induced strain formed by a simple and inexpensive chemical procedure has a similar effect to that of strain at a hetero-interface originated from lattice mismatch. These findings provide novel insights into strain engineering.

\section{Disclosures}

The authors have nothing to disclose. 


\section{Acknowledgements}

N. S. was financially supported by the Japan Society for the Promotion of Science (JSPS) Grant-in-Aid for Scientific Research (KAKENHI) (Grant No. 26810126). Y. Y. is grateful to the Deanship of Scientific Research, King Saud University for founding through Vice Deanship of Scientific Research Chairs.

Wide-angle XRD measurements were conducted at the Nano-Processing Facility, supported by the Innovation-Boosting Equipment Common (IBEC) Innovation Platform, National Institute for Advanced Industrial Science and Technology (AIST), Japan. Raman spectra measurement and TEM observation of the porous thin film was conducted by HORIBA TECHNO SERVICE Co., Ltd. and Foundation for Promotion of Material Science and Technology of Japan (MST), respectively. MST also conducted visualization of the strain from TEM images. We thank Andrew Jackson, PhD, from Edanz Group (www.edanzediting.com/ac) for editing a draft of this manuscript.

\section{References}

1. Choi, K. J. et al. Enhancement of Ferroelectricity in Strained $\mathrm{BaTiO}_{3}$ Thin Films. Science. 306 (5698), 1005-1009 (2004).

2. Nagarajan, V. et al. Misfit dislocation in nanoscale ferroelectric heterostructures. Appl. Phys. Lett. 86 (19), 192910 (2005).

3. Wimbush, S. C. et al. Interfacial Strain-Induced Oxygen Disorder as the Cause of Enhanced Critical Current Density in Superconducting Thin Films. Adv. Funct. Mater. 19 (6), 835-841 (2009).

4. Harrington, S. A. et al. Thick lead-free ferroelectric films with high Curie temperatures through nanocomposite-induced strain. Nat. Nanotechnol. 6 (8), 491-495 (2011).

5. Suzuki, N. et al., Synthesis of Highly Strained Mesostructured $\mathrm{SrTiO}_{3} / \mathrm{BaTiO}_{3}$ Composite Films with Robust Ferroelectricity. Chem. -Eur. J. 19 (14), 4446-4450 (2014).

6. Kresge, C. T., Leonowicz, M. E., Roth, W. J., Vartuli, J. C., Beck, J. S. Ordered mesoporous molecular sieves synthesized by a liquid-crystal template mechanism. Nature. 359 (6397), 710-712 (1992).

7. Yang, P., Zhao, D., Margolese, D. I., Chmelka, B. F., Stucky, G. D. Generalized syntheses of large-pore mesoporous metal oxides with semicrystalline frameworks. Nature. 396 (6707), 152-155 (1998).

8. Suzuki, N., Jiang, X., Salunkhe, R. R., Osada, M., Yamauchi, Y. Chemical Preparation of Ferroelectric Mesoporous Barium Titanate Thin Films: Drastic Enhancement of Curie Temperature Induced by Mesopore-Drived Strain. Chem. -Eur. J. 20 (36), 11283-11286 (2014).

9. Tenne, D. A., Xi, X. Raman Spectroscopy of Ferroelectric Thin Films and Superlattices. J. Am. Ceram. Soc. 91 (6), 1820-1834 (2008).

10. Ide, T., Sakai, A., Shimizu, K. Nanometer-Scale Imaging of Lattice Deformation with Transmission Electron Micrograph. Jpn. J. Appl. Phys., Part 2. 37 (12B), L1546-L1548 (1998).

11. Hoshina, T., Wada, S., Kuroiwa, Y., Tsurumi, T. Composite structure and size effect of barium titanate nanoparticles. Appl. Phys. Lett. 93 (19) 192914 (2008).

12. Feng, C., Zhou, D. X., Gong, S. P. Core-shell structure and size effect in barium titanate nanoparticle. Phys. B. 406 (6-7), 1317-1322 (2011).

13. Suzuki, N. et al. Origin of thermally stable ferroelectricity in a porous barium titanium thin film synthesized through block copolymer templateing. APL Mater. 5 (7), 076111 (2017). 